\title{
The eddy heat-flux in rotating turbulent convection
}

\author{
G. Rüdiger ${ }^{1}$, P. Egorov ${ }^{1}$, L. L. Kitchatinov ${ }^{1,2}$, and M. Küker ${ }^{1}$ \\ 1 Astrophysikalisches Institut Potsdam, An der Sternwarte 16, 14482, Potsdam, Germany \\ e-mail: gruediger@aip.de \\ 2 Institute for Solar-Terrestrial Physics, PO Box 4026, Irkutsk 664033, Russia
}

Received 15 July 2004 / Accepted 18 October 2004

\begin{abstract}
The three components of the heat-flux vector $\boldsymbol{F}=\rho C_{\mathrm{p}}\left\langle\boldsymbol{u}^{\prime} T^{\prime}\right\rangle$ are numerically computed for a stratified rotating turbulent convection using the NIRVANA code in a flat box. The latitudinal component $F_{\theta}$ proves to be negative (positive) in the northern (southern) hemisphere so that the heat always flows towards the poles. As a surprise, the radial heat-flux $F_{r}$ peaks at the equator rather than at the poles (Taylor numbers $O\left(10^{6}\right)$ ). The same behavior is observed for the radial turbulence intensity $\left\langle u_{r}^{\prime 2}\right\rangle$ which for free turbulence is also believed to peak at the poles (see Eq. (19) below). As we can show, however, the consequences of this unexpected result (also obtained by Käpylä et al. 2004, A\&A, 422, 793) for the theory of differential rotation are small as mainly the $F_{\theta}$ is responsible to solve the "Taylor number puzzle".

In all our simulations the azimuthal component $F_{\phi}$ proves to be negative so that the rotating turbulence produces a westwards directed azimuthal heat-flux which should be observable. Fluctuations with higher temperature are expected to be anticorrelated with their own angular velocity fluctuations. We find this rotation-induced result as understandable as the $F_{\phi}$ is closely related to the radial $\Lambda$-effect which is known to be also negative in stratified and rapidly rotating convection zones.
\end{abstract}

Key words. convection - turbulence - Sun: granulation - Sun: rotation

\section{Motivation}

There are more and more data describing the surface rotation law of stars, the majority of which complies both with the sign and amplitude of the known equatorial acceleration of the Sun. In order to compare the various observational results a relation

$\Omega_{\mathrm{eq}}-\Omega_{\mathrm{pole}} \propto \Omega_{\mathrm{eq}}^{n^{\prime \prime}}$

has been introduced with $n^{\prime \prime}$ representing the $\Omega$-dependence of the equator-pole difference of the surface angular velocity. It proved to be weak in the first papers in this research $\left(n^{\prime \prime} \simeq 0.15\right.$, Hall 1991) while in recent studies values of 0.58 (Messina \& Guinan 2003) and 0.66 (Reiners \& Schmitt 2003) have been found. A rotational influence upon the rotation law is clearly existing but it seems not to be too strong.

To find the influence of the global rotation on the turbulent convection in the stellar convection zones is thus the basic problem of the theory of differential rotation. The resulting flow pattern ("rotating turbulent convection") simultaneously transports both the heat and the angular momentum. The resulting angular momentum transport has been described with the $\Lambda$-effect whose properties easily can be reproduced with box simulations (see the references in Rüdiger \& Hollerbach 2004). In the present paper it is the eddy heat-flux in rotating turbulent convection which shall be rediscussed here by means of new box simulations.
For the usual Boussinesq relation

$\boldsymbol{F}=-\rho T \chi \nabla S$

between the eddy heat-flux and the entropy gradient Weiss (1965) and Durney \& Roxburgh (1971) parameterized the influence of a global rotation by

$\chi=\chi_{0}\left(1+\varepsilon \cos ^{2} \theta\right)$

with $\varepsilon$ representing the rotational influence. The eddy conductivity is assumed to involve the main influence of the turbulent flow pattern which due to the basic rotation strongly depends on the colatitude $\theta$. The ansatz (3) leads to a $\theta$-dependence of all thermodynamic quantities with a temperature difference between pole and equator so that a meridional flow is the immediate consequence. There was no possibility at that time for a detailed theory of the connection of $\chi_{0}$ and $\varepsilon$ with the characteristic parameters of the turbulence (see also Belvedere et al. 1980).

Meridional flows are playing a more and more important role in the solar (magneto-)hydrodynamics. The theory of the differential rotation in the convection zone and in the tachocline as well as the theory of the solar dynamo are important examples. This paper discusses details of the thermodynamics of the rotation theory. We shall present the ideas for the construction of the eddy-conductivity tensor in the next section. In the following sections the resulting analytical but quasilinear findings 
will be compared with the results of fully nonlinear simulations with the finite-difference code NIRVANA which solves the time-dependent nonrelativistic MHD equations for thermodynamic equilibrium in three dimensions (see Ziegler 1998, 1999).

\section{Quasilinear theory}

In rotating turbulent fluids, the relation between the turbulent heat-flux $\boldsymbol{F}=\rho C_{\mathrm{p}}\left\langle\boldsymbol{u}^{\prime} T^{\prime}\right\rangle$ and the superadiabatic temperature gradient

$\boldsymbol{\beta}=\frac{\boldsymbol{g}}{C_{\mathrm{p}}}-\nabla T$

( $\boldsymbol{g}$ is gravity) is a tensorial one, i.e.

$F_{i}=\rho C_{\mathrm{p}} \chi_{i j} \beta_{j}$

with $\beta=\nabla T^{\text {ad }}-\nabla T=-\left(T / C_{\mathrm{p}}\right) \nabla S$, where $S$ is the specific entropy. In the simplest case known from the mixing-length theory it is $\chi_{i j}=\chi_{\mathrm{T}} \delta_{i j}$ so that Eq. (2) results (instead of $\boldsymbol{F}=$ $-\rho C_{\mathrm{p}} \chi_{\mathrm{T}} \nabla T$ for incompressible fluids).

\subsection{Isotropic turbulence subject to rotation}

For rotating fluids without any other preferred direction than the rotation vector $\Omega$ it is simply

$\chi_{i j}=\chi_{\mathrm{T}} \delta_{i j}+\chi_{\|} \Omega_{i} \Omega_{j}+\tilde{\chi} \epsilon_{i p j} \Omega_{p}$

(Kitchatinov et al. 1994; Canuto \& Minotti 2001; Canuto 2002). Obviously, by the last (antisymmetric) term in Eq. (6) a heat-flux in the azimuthal direction is described which already has been discussed by Gough (1978), Rüdiger (1989) and Canuto (2002, see his Eq. (13)). Our simulations presented in the Sect. 6 will show that this term does indeed exist and it is strongly related to the angular momentum transport in rotating convection zones.

The poloidal components of Eq. (6) in spherical coordinates are

$\chi_{r r}=\chi_{\mathrm{T}}+\cos ^{2} \theta \chi_{\|} \Omega^{2}$,

$\chi_{\theta r}=-\sin \theta \cos \theta \chi_{\|} \Omega^{2}$.

If the averages are taken over the horizontal plane, $\beta$ has only a radial positive component which is positive in the convectively unstable zones.

With Eq. (7) the heat-transport in rotating but otherwise isotropic turbulence is rather simple. If the latitudinal heattransport goes to the poles (equator) then the radial heattransport at the poles is always stronger (weaker) than at the equator. In the first case the $\chi_{\|}$is positive and in the second case it is negative.

The coefficients $\chi_{\mathrm{T}}$ and $\chi_{\|}$have been computed by Kitchatinov et al. (1994, their Fig. 1). They both are positive. The $\chi_{\mathrm{T}}$ vanishes like $O\left(\Omega^{-1}\right)$ for fast rotation while the $\chi_{\|}$behaves like $O\left(\Omega^{-3}\right)$. Hence, for isotropic turbulence under the influence of a global rotation, the radial eddy heat-flux peaks at the poles, and the latitudinal heat-flux goes to the poles.

\subsection{Anisotropic turbulence subject to rotation}

The situation is more complicated if the turbulence-field without rotation is already anisotropic. The anisotropy (unit) vector may be $\boldsymbol{G}$. In this case extra terms appear in Eq. (6) which in the simplest case run with the second order in $\boldsymbol{G}$, i.e.

$\chi_{i j}=\ldots+\chi_{1}(\boldsymbol{G} \boldsymbol{\Omega})^{2} \delta_{i j}+\chi_{2}(\boldsymbol{G} \boldsymbol{\Omega})\left(G_{i} \Omega_{j}+G_{j} \Omega_{i}\right)$.

These terms provide the new contributions

$\chi_{r r}=\ldots+\left(\chi_{1}+2 \chi_{2}\right) \cos ^{2} \theta \Omega^{2}$

$\chi_{\theta r}=\ldots-\chi_{2} \sin \theta \cos \theta \Omega^{2}$.

To the poloidal components (7) of the heat-conductivity tensor. Hence, now

$\chi_{r r}=\chi_{\mathrm{T}}+\left(\hat{\chi}+\chi_{\|}\right) \Omega^{2} \cos ^{2} \theta$,

$\chi_{\theta r}=-\chi_{\|} \Omega^{2} \sin \theta \cos \theta$,

where some of the notations have slightly been changed. The sign of the unknown quantity $\hat{\chi}$ may fix the latitudinal profile of the radial heat-flux.

In the following we shall compute (10) for various rotation rates by means of numerical box simulations. We shall find, indeed, that the pole-equator difference of the radial heat-flux is a sensitive function of rotation, stratification and/or boundary conditions. This is not true, however, for the latitudinal heat-flux which will prove to be towards the poles in all our simulations.

\subsection{Rotating free turbulence}

There is a close relation of the heat-flux tensor and the onepoint correlation tensor

$Q_{i j}=\left\langle u_{i}^{\prime}(\boldsymbol{x}, t) u_{j}^{\prime}(\boldsymbol{x}, t)\right\rangle$.

In order to demonstrate this basic outline we start from the quasilinear relation

$$
\begin{aligned}
\chi_{i j} & =\int \frac{\hat{Q}_{i j}(\boldsymbol{k}, \omega)}{-\mathrm{i} \omega+\chi k^{2}} \mathrm{~d} \boldsymbol{k} \mathrm{d} \omega \\
& =\int \frac{\chi k^{2} \hat{Q}_{i j} \mathrm{~d} \boldsymbol{k} \mathrm{d} \omega}{\omega^{2}+\chi^{2} k^{4}}+\mathrm{i} \int \frac{\omega \hat{Q}_{i j} \mathrm{~d} \boldsymbol{k} \mathrm{d} \omega}{\omega^{2}+\chi^{2} k^{4}}
\end{aligned}
$$

between the spectral tensor $\hat{Q}_{i j}$ of the turbulence and the heatconductivity tensor $\chi_{i j}$ (e.g. Rüdiger 1989). The last term in Eq. (12) provides an antisymmetric part of the $\chi_{i j}$-tensor which is still ignored.

In the remaining term for $\chi \rightarrow 0$ a Dirac $\delta$-function is involved so that in this approximation

$\chi_{i j}=\pi \int \hat{Q}_{i j}(k, 0) \mathrm{d} k \equiv \frac{1}{2} \int Q_{i j}(0, \tau) \mathrm{d} \tau$.

If this relation is applied and if the $\tau$-integral is approximated by $\tau_{\text {corr }}$ then

$\chi_{i j}=\frac{1}{2} \tau_{\text {corr }} Q_{i j}$.

It makes sense, therefore, to discuss briefly the one-point correlation tensor $Q_{i j}$ under the influence of rotation. In the 
Appendix the over-all structure of this tensor is given within the $\tau$-approximation which is adopted in the following. Two properties are particularly stressed here: i) the anisotropy of the rotating turbulence at the poles; and ii) the pole-equator difference of the radial turbulence intensity $\left\langle u_{r}^{\prime 2}\right\rangle$.

At the poles we have $\left\langle u_{\theta}^{\prime 2}\right\rangle=\left\langle u_{\phi}^{\prime 2}\right\rangle$. For the characterization of the anisotropy it is thus enough to write

$$
\begin{gathered}
\left\langle u_{r}^{\prime 2}\right\rangle-\left\langle u_{\theta}^{\prime 2}\right\rangle-\left\langle u_{\phi}^{\prime 2}\right\rangle=\left\langle u_{r}^{\prime 2}\right\rangle-2\left\langle u_{\phi}^{\prime 2}\right\rangle= \\
\left(\phi_{\|}-\phi+a\left(\phi_{1}+\phi_{2}-2 \phi_{3}-\phi^{\prime}\right)\right)\left\langle u_{r}^{(0) 2}\right\rangle
\end{gathered}
$$

with $a$ after Eq. (A.4). This anisotropy will be considered in this section exclusively. The azimuthal heat-flux is discussed in Sect. 6. With the relations (A.11) it follows for fast rotation that

$\left\langle u_{r}^{\prime 2}\right\rangle-\left\langle u_{\theta}^{\prime 2}\right\rangle-\left\langle u_{\phi}^{\prime 2}\right\rangle=O\left(\Omega^{*-3}\right)$.

Here

$\Omega^{*}=2 \tau_{\text {corr }} \Omega$

is the Coriolis number (or inverse Rossby number) of the turbulence. Hence,

$\left\langle u_{r}^{\prime 2}\right\rangle \simeq\left\langle u_{\phi}^{\prime 2}\right\rangle+\left\langle u_{\theta}^{\prime 2}\right\rangle$.

At the poles, the fast rotation results in a dominance of the vertical turbulence components.

Canuto et al. (1994) have demonstrated with their rather general model how the global rotation influences the formation of anisotropy between the components of the turbulence intensity. If in the equatorial plane without rotation both the turbulent intensities $\left\langle u_{r}^{\prime 2}\right\rangle$ and $\left\langle u_{\phi}^{\prime 2}\right\rangle$ strongly differ then the rotation is smoothing the differences and may even completely suppress them (Rüdiger et al. 2002). For faster rotation there is thus a clear tendency for a return-to-isotropy. A similar (even crossover) behavior for anisotropic turbulence has also been found for his rotating convection-turbulence by Chan (2001).

For the radial turbulence intensity one finds the relation

$\left.\left\langle u_{r}^{\prime 2}\right\rangle\right|_{\text {pole }}-\left.\left\langle u_{r}^{\prime 2}\right\rangle\right|_{\text {eq }}=\left(\phi_{\|}+a\left(\phi_{\|}^{\prime}+\phi_{1}\right)\right)\left\langle u_{r}^{(0) 2}\right\rangle$.

The contributions of the isotropic and anisotropic parts of turbulence to the pole-equator difference (19) are shown in Fig. 1 as functions of the Coriolis number. For radial-dominated free turbulence $(a<0)$ the expression (19) is always positive. Therefore, $\left\langle u_{r}^{\prime 2}\right\rangle$ for rotating turbulence should be always greater at the poles rather than at the equator.

\section{Basic equations, the model}

3D numerical simulations of compressible, thermal convection under the influence of rotation are made with the finitedifference, fractional-step code NIRVANA (Ziegler 1998, 1999) in a small rectangular box defined on a Cartesian grid. It can be considered as a small piece of a spherical shell. The domain is placed tangentially at different colatitudes from $\theta=0^{\circ}$ (at the north pole) to $180^{\circ}$ (at the south pole) with a step of $30^{\circ}$,

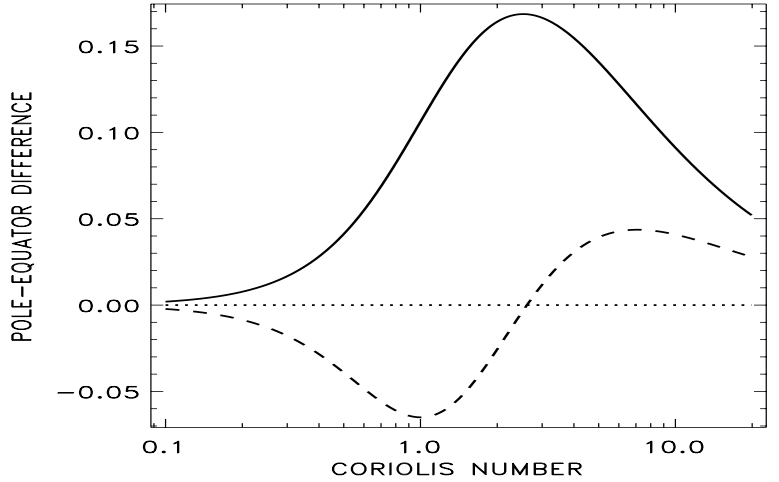

Fig. 1. Contributions of the isotropic (full line) and anisotropic (dashed line) parts of turbulence into the normalized pole-equator difference (19) of the radial turbulence intensity. The full and dashed lines are the functions $\phi_{\|}$and $\phi_{\|}^{\prime}+\phi_{1}$.

and contains a convectively unstable layer, surrounded by stable stratified layers with overshooting convection. The height of the convection zone $d$ is chosen as the unit length.

The box rotates around the polar axis from west to east (the angular velocity vector $\boldsymbol{\Omega}$ points toward the north pole). The geometry of the computational domain is $(x, y, z) \in[0,8 d] \in$ $[0,8 d] \in[-2 d, 0]$. This volume is discretized by $100 \times 100 \times$ 128 grid points which are uniformly distributed in each coordinate direction.

The governing equations describing thermal convection in a rotating stratified medium are

$\frac{\partial \rho}{\partial t}=-\nabla \cdot(\rho \boldsymbol{u})$

$\frac{\partial \rho \boldsymbol{u}}{\partial t}=-\nabla \cdot(\rho \boldsymbol{u} \boldsymbol{u})-\nabla P+\nabla \cdot \pi+\rho \boldsymbol{g}-2 \rho \boldsymbol{\Omega} \times \boldsymbol{u}$,

$\frac{\partial \rho U}{\partial t}=-\nabla \cdot(\rho U \boldsymbol{u})-P \nabla \cdot \boldsymbol{u}+Q_{\mathrm{vis}}+\nabla \cdot\left(\rho C_{\mathrm{p}} \chi \nabla T\right)$.

The notation for physical variables is standard ( $U$ is thermal energy density, $\pi$ is the viscous stress tensor and $Q_{\text {vis }}$ is the viscous heating term.

The Eqs. (20)-(22) are closed through the ideal gas equation $P=(\mathcal{R} / \mu) \rho T$. The initial distribution of the physical quantities represents a 3-layer polytrophic stratification. We assume all quantities to be periodic in the horizontal directions. At the bottom $(z=-2)$ and top $(z=0)$ of the box impermeable conditions are imposed for the vertical velocity, while the horizontal velocities satisfy stress-free boundary conditions. The temperature and density are fixed at the top of the domain and a constant heat-flux is injected at the bottom.

The dimensionless parameters $\mathrm{Ra}$, Pr and $\mathrm{Ta}$ are used to control the simulations. In our calculations $\mathrm{Ra}=3 \times 10^{5}$ and $\operatorname{Pr}=0.1$, while Ta $\in\left\{10^{5}, 10^{6}\right\}$.

\section{Rotation-induced anisotropic turbulence}

We start with a discussion of the basic anisotropy between vertical and horizontal turbulence intensities without (Fig. 2, left) and with rotation (Fig. 2, middle \& right). Without rotation except in the top layer the turbulence is vertically dominated (Fig. 2, left). As it must be, both the horizontal 

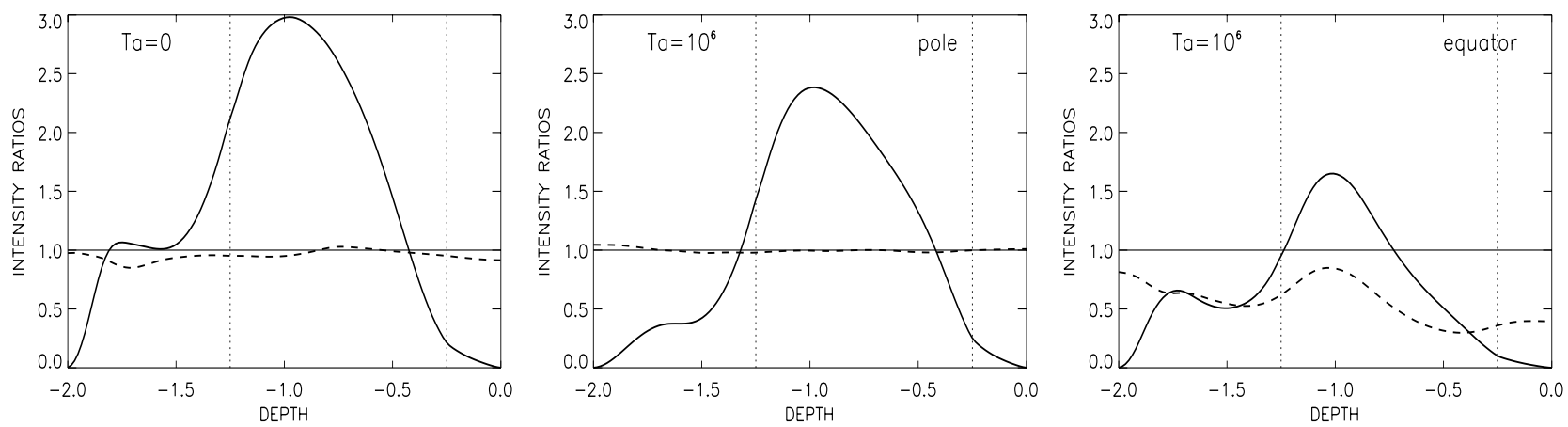

Fig. 2. Ratios of the turbulence intensity for the nonrotating and rotating turbulence fields. Solid: $\left\langle u_{r}^{\prime 2}\right\rangle /\left\langle u_{\phi}^{\prime 2}\right\rangle$, dashed: $\left\langle u_{\theta}^{\prime 2}\right\rangle /\left\langle u_{\phi}^{\prime 2}\right\rangle$. Left: except the surface layers the turbulence is strongly vertical-dominated. It is even isotropic in the lower overshoot region. Middle: Ta $=10^{6}$, pole. Right: $\mathrm{Ta}=10^{6}$, equator. Under the influence of rotation only the lower half of the box remains vertical-dominated. Note that the basic rotation originates a (mild) dominance of the horizontal motions over the radial motions in the lower overshoot region.

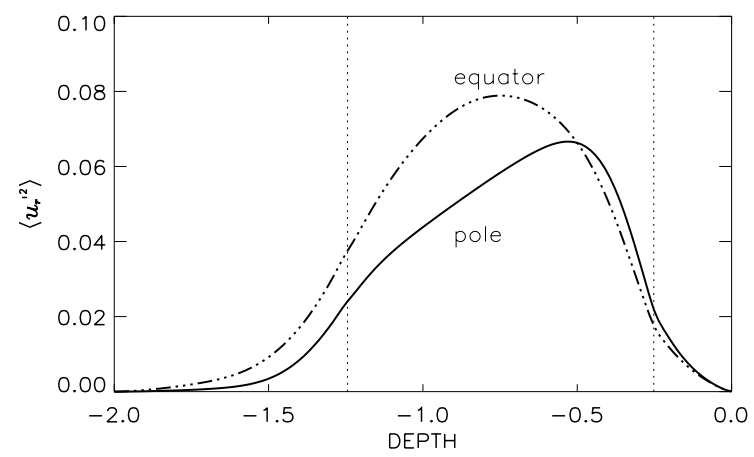

Fig. 3. The radial turbulence intensity for $\mathrm{Ta}=10^{6}$. Note that except the top layer the turbulence at the equator exceeds the polar values. Quite a similar result follows from box simulations without any density stratification (Giesecke et al. 2004).

intensities are equal. The same is true for $\mathrm{Ta}=10^{6}$ in the polar region (Fig. 2, middle). For the equator, however, the vertical-horizontal anisotropy is more and more reduced but a new anisotropy develops between both the horizontal components (Fig. 2, right).

The simulations also provide the anisotropies in the overshoot region. This is in particular important for the lower overshoot region in relation to the tachocline discussion. It has been argued that the stability of this zones against convection changes the turbulence to the horizontal-type (Spiegel \& Zahn 1992). This is not observable in Fig. 2 for the case without rotation (left).

The behavior of the vertical turbulence intensity is even more important. For free and anisotropic turbulence we expect for vertically-dominated turbulence that the polar values exceed the equatorial values (see Appendix). The opposite behavior is shown by the simulations. Figure 3 reveals that in the bulk of the convection box the equator dominates the poles, i.e.

$\left.\left.\left\langle u_{r}^{\prime 2}\right\rangle\right|_{\mathrm{eq}}\right\rangle\left.\left\langle u_{r}^{\prime 2}\right\rangle\right|_{\text {pole }}$.

This unexpected result (see Eq. (19)) has important consequences for the meridional components of the heat-flux. After Eq. (13) the heat-conductivity tensor is proportionate to the one-point correlation tensor (multiplied with a correlation
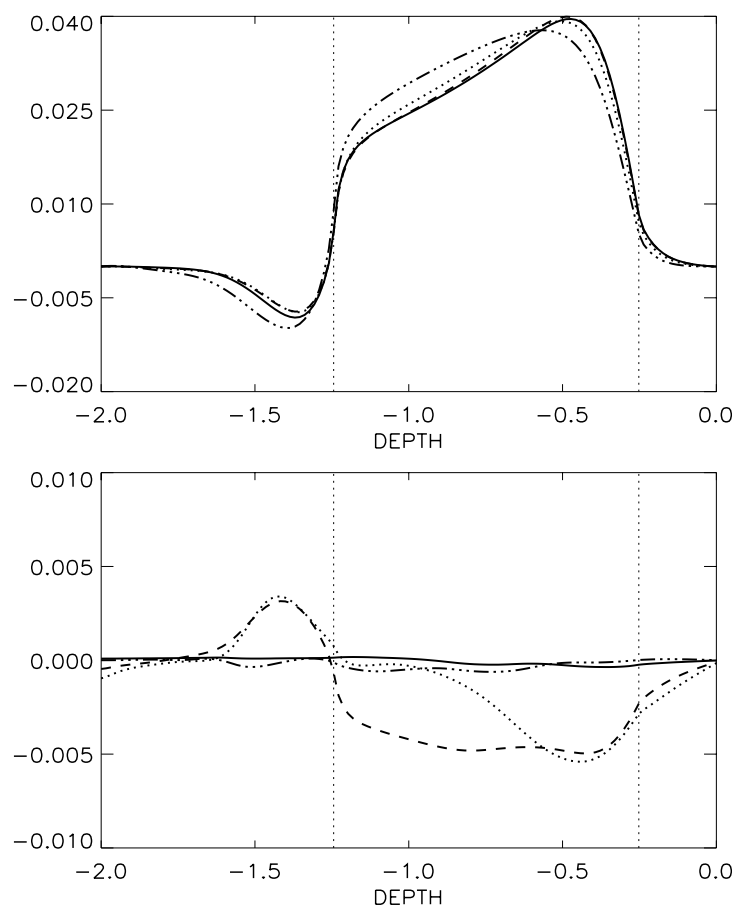

Fig. 4. The correlations $\left\langle\boldsymbol{u}^{\prime} T^{\prime}\right\rangle$ for different colatitudes in the box simulations after horizontally and time-averaging vs. depth, $\mathrm{Ta}=10^{6}$. Top: $\left\langle u_{r}^{\prime} T^{\prime}\right\rangle$, bottom: $\left\langle u_{\theta}^{\prime} T^{\prime}\right\rangle$. Solid: pole, dashed: $30^{\circ}$, dotted: $60^{\circ}$, triple-dot-dashed: equator.

time) so that it should not be too surprising if also the radial heat-flux $F_{r}$ would peak at the equator rather than at the poles. The simulations confirm this expectation.

\section{The poloidal heat-flux}

Figure 4 (top) shows the depth-profile of the correlation $\left\langle u_{r}^{\prime} T^{\prime}\right\rangle$ in the box for various latitudes. Due to the rotation, the values differ between poles and the equator. The pole-equator difference in the radial heat-flux depends, however, on the radius. Except for the top layer, the eddy heat-flux at the equator exceeds the eddy heat-flux at the poles. In the top layers, where after Fig. 2 the turbulence is horizontally-dominated, the polar heat-flux dominates the equatorial one. 


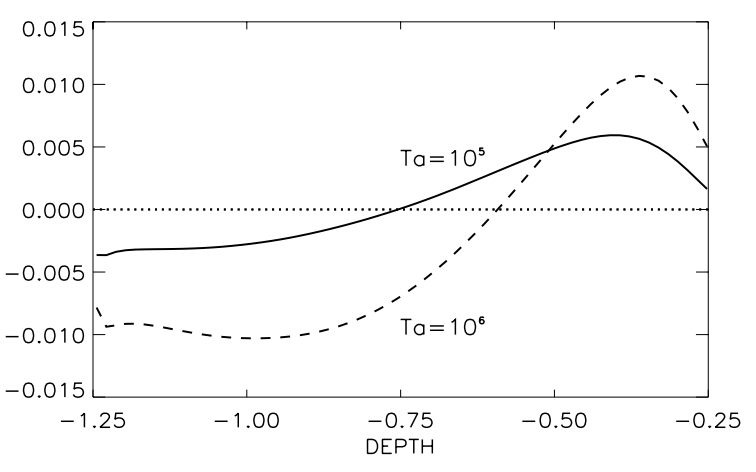

Fig. 5. The coefficient function $\hat{\chi}$ in the box for different Taylor numbers. Note the characteristic zero close to the top of the convective box.

This is a characteristic but unexpected result. It does not contradict, however, the findings of Tilgner \& Busse (1997, their Fig. 8) in which the latitudinal profiles of the radial heatflux differ for differing parameters. For the cases with large Pr at the inner boundary the heat-flux dominates indeed at the equator; and at the outer boundary the heat-flux dominates indeed at the poles.

Also Rieutord et al. (1994, their Fig. 8a) and Käpylä et al. (2004, their Fig. 7) found similar results. Here we are led to the general conclusion that a crossover exists of the poleequator difference of the radial eddy heat-flux almost at the same depth where the vertically-dominated turbulence changes to a horizontally-dominated turbulence. As we have demonstrated with Eq. (14) the behavior of the radial heat-flux is a direct reflection of the rotation-influenced radial turbulence intensity $\left\langle u_{r}^{\prime 2}\right\rangle$. It is shown in Fig. 3 that in the box (except the outermost layer) the $\left\langle u_{r}^{\prime 2}\right\rangle$ at the equator exceeds the value at the poles.

A similar crossover does not exist for the latitudinal eddy heat-flux $\left\langle u_{\theta}^{\prime} T^{\prime}\right\rangle$ plotted in Fig. 4 (bottom). This heat-flux vanishes by definition at the poles and the equator. Between these extrema the heat flows towards the pole in the convection zone (and towards the equator in the lower overshoot region). It is a consequence of the Coriolis force which is not involved in the description (2). The function $\chi_{\|}$in $(10)_{2}$, obviously, is positivedefinite as was predicted in Sect. 2.1 by a much simpler consideration.

\section{The azimuthal heat-flux}

Obviously, the last term in Eq. (6) is linear in $\Omega$ in opposition to the second one which only appears in the second order in $\Omega$. The expression linear in $\Omega$ leads to an azimuthal component of the heat-flux of

$F_{\phi}=\rho C_{\mathrm{p}} \chi_{\phi r} \beta_{r} \Omega$,

where $\beta_{r}>0$ in convection zones. The sign of $\chi_{\phi r}$, therefore, determines the sign of $F_{\phi}$. One could argue, however, that for axisymmetric constellations the $F_{\phi}$ is not important because of $\operatorname{div} \boldsymbol{F}=0$ in that case but i) many stars are not axisymmetric; and ii) the linear-in- $\Omega$ term $F_{\phi}$ should be important for the comparison of (quasilinear) theory and (nonlinear) simulation. If the rather simple analytical theory of $F_{\phi}$ cannot be

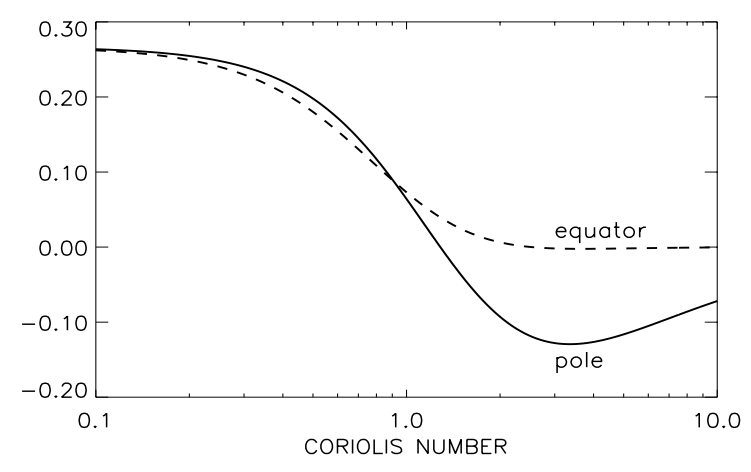

Fig. 6. The $\Lambda$-effect quantity $V$ as a function of latitude and Coriolis number (i.e. $\Omega$ ). It is mainly negative between pole and equator. The turbulence model is highly density-stratified.

confirmed by numerical simulations then the chance is small to understand the higher-order terms in Eq. (6) which concern the poloidal components of the vector $\boldsymbol{F}$.

The azimuthal heat-flux results from the relation

$\chi_{\phi r}=\sin \theta \Omega \tilde{\chi}$.

The quantity $\tilde{\chi}$ in Eq. (25) has been computed for a simple turbulence model subject to a basic rotation but without stratification by Kitchatinov et al. (1994). The term was positivedefinite but it proved to be very small. The main reason for its smallness is that the integral kernel runs with $\omega^{2}$ so that in the $\tau$-approximation the term vanishes.

This is not true if anisotropic turbulence is considered. After Eq. (14) we have

$\chi_{\phi r}=\frac{1}{2} \tau_{\mathrm{corr}} Q_{\phi r}$.

As known the zonal cross correlation $Q_{\phi r}$ only exists for rotating stratified turbulence and we write

$Q_{\phi r}=v_{\mathrm{T}} \Omega V \sin \theta$

with $V$ as the vertical component of the $\Lambda$-effect. Along this way we arrive at

$\chi_{\phi r}=\frac{\Omega^{*}}{4} \nu_{\mathrm{T}} V \sin \theta$.

The zonal heat-flux (24) should thus be positive (negative) for positive (negative) $V$. A very similar relation has been formulated by Gough (1978) and one can also find such terms in the expressions given by Canuto (2002). Obviously, the $F_{\phi}$ and the radial $\Lambda$-effect have the same sign. In Fig. 6 the radial $\Lambda$-effect quantity $V$ is given which was obtained by Kitchatinov $\&$ Rüdiger (1993) within the quasilinear $\tau$-approximation. Generally, the function $V$ is negative which result has recently been confirmed numerically by Chan (2001) and Käpylä et al. (2004). Hence, the $F_{\phi}$ should be negative and this is also the result of the simulations shown in Fig. 7. Positive (negative) fluctuations $u_{\phi}^{\prime}$ are thus be correlated with negative (positive) temperature fluctuations which should be observable. 


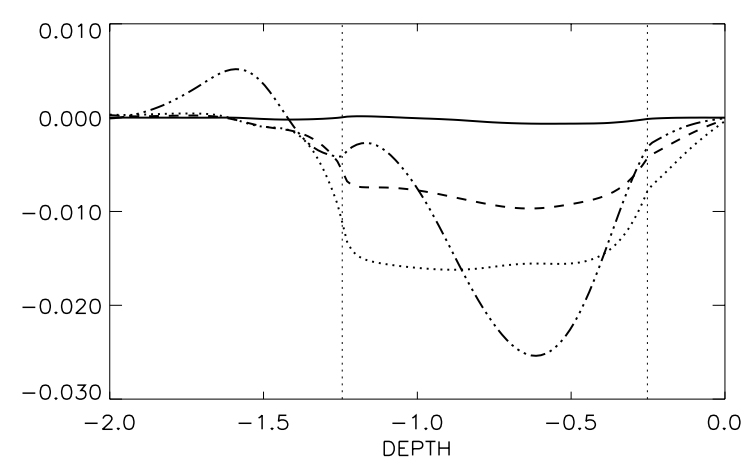

Fig. 7. The correlation $\left\langle u_{\phi}{ }^{\prime} T^{\prime}\right\rangle$ for different colatitudes in the box simulations after horizontally and time-averaging vs. depth, $\mathrm{Ta}=10^{6}$.

\section{Rotation-law consequences}

As mentioned in Sect. 1 the rotation laws which have been computed so far (Kitchatinov \& Rüdiger 1999; Küker \& Stix 2001) were obtained with a radial heat-flux which peaks at the poles as the result of the basic rotation. The simulations do not confirm this latitudinal profile. On the other hand, the rotation laws are computed with a latitudinal heat-flux which goes to the poles and this is confirmed by the simulations. We have thus to check the consequences of the new situation for the rotation law theory.

As a demonstration of the complex character of the resulting mean-field rotation laws in Fig. 8 (top) the rotation law is given as due to the $\Lambda$-effect alone (details given by Küker \& Stix 2001) without both meridional flow and rotation-induced eddy heat-flux. The rotation profile well complies with the observations (see Küker et al. 1993).

The inclusion of the meridional flow (Fig. 8, bottom) drastically changes the situation. The resulting poleward surface flow strongly reduces the equator-pole difference and according to the Taylor-Proudman theorem the $\Omega$-isolines become parallel to the rotation axis. In this case one finds a slight superrotation beneath the equator and a rather uniform angular velocity beneath the pole - in great contrast to the observations.

The situation changes with the rotation-induced eddy heatflux included. The poles become warm and a circulation develops towards the equator. Hence, the total meridional flow becomes rather slow and again we have the situation of Fig. 8 (top). This is the solution of the "Taylor number puzzle" which consists in the existence of two opposite directions of the meridional circulation (Fig. 9, top). In Fig. 9 (bottom) the numerical experiment with (artificially) $\chi_{\theta r}=0$ reveals that it is the $\chi_{\theta r}$-effect which solves the Taylor number puzzle rather than the latitude-profile of $\chi_{r r}$.

\section{Discussion}

For rotating free anisotropic turbulence Eq. (19) provides a dominance of the vertical turbulence intensity at the poles. Our box simulations reveal the opposite behavior. As shown in Fig. 3 the turbulence at the equator dominates. This is not a consequence of the density stratification which was not involved in the evaluation of the correlation tensor for rotating
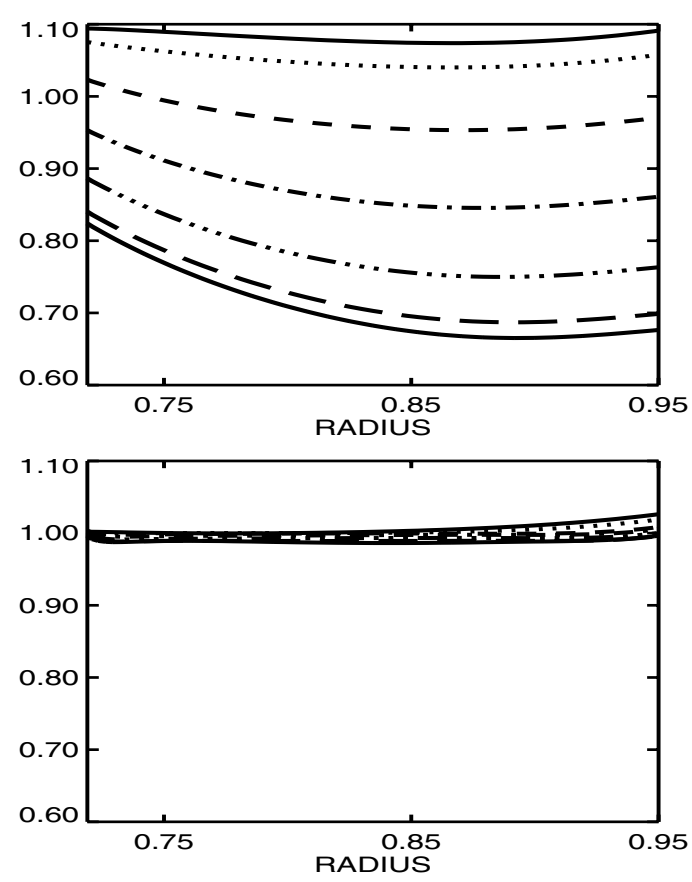

Fig. 8. The rotation law in the solar convection zone without the influence of the rotation-modified heat-flux without (top) and with (bottom) meridional circulation.
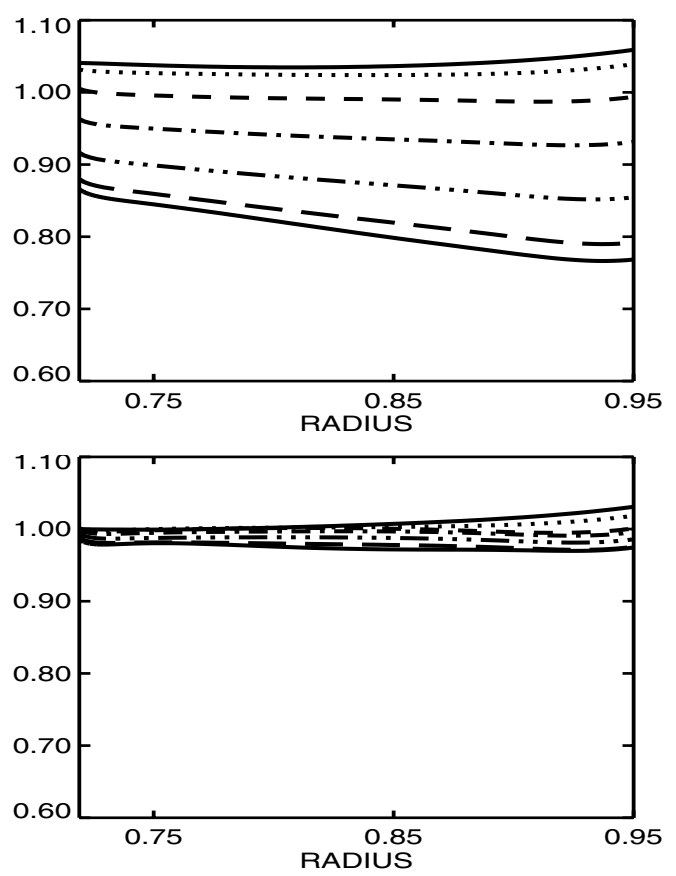

Fig. 9. The same as in Fig. 8 but with the full rotation-modified heatflux (top) and $\chi_{\theta r}=0$ (see Eq. $(10)_{2}$ bottom). Obviously, the rotationinduced off-diagonal component $\chi_{\theta r}$ produces the main effects.

free turbulence as given in the Appendix. Giesecke et al. (2004) considered boxes without any density stratification but with the same result. One needs global simulations in order to find out whether or not the limited size of the boxes is responsible for the unexpected effect.

An immediate consequence of this result concerns the latitude-dependence of the vertical turbulent heat-transport which is given in Fig. 4 (top). In the bulk of the box, the 
heat-flux at the equator exceeds the vertical heat-flux at the poles $^{1}$. For free turbulence the quasilinear theory on the basis of the $\tau$-approximation leads to the opposite behavior. There is possibly a simple explanation of the differences for the latitudinal profile of $\left\langle u_{r}^{\prime 2}\right\rangle$ for free turbulence and for box simulations. For rapid rotation the Taylor-Proudman theorem strongly damps the $u_{r}$-component of the turbulence at the poles due to the boundary condition $u_{r}^{\prime}=0$ at the top and bottom of the box.

This argument hardly holds for the cross correlations $\left\langle u_{\theta}^{\prime} u_{r}^{\prime}\right\rangle$ and $\left\langle u_{\phi}^{\prime} u_{r}^{\prime}\right\rangle$ which identically vanish at the poles (see Eqs. (A.12) and (A.13)). The results of the simulations for the eddy heat-flux components $F_{\theta}$ and $F_{\phi}$ do fully comply with the analytical quasilinear expressions. Both correlations are negative at the northern hemisphere ${ }^{2}$. The latitudinal heat transport is therefore always poleward and the azimuthal heat-flux is always westwards. The results of Käpylä et al. (2004) for the latitudinal heat-transport are here less coherent.

The correlation $\left\langle u_{\phi}^{\prime} T^{\prime}\right\rangle$ is due to the rotational influence and it represents the radial angular momentum transport by the turbulence. It should be observable at the solar surface. Convective-originated temperature fluctuations like those of the mesogranulation are expected to be anticorrelated with their local angular velocity fluctuations (see Duvall \& Gizon 2000).

For axisymmetric stars, of course, the longitudinal heatflux is not important because of $\partial F_{\phi} / \partial \phi=0$ but this might not to be true for close binaries or also for single stars with distinct nonaxisymmetric surface structures like the (flip-flop) FK Com stars.

More clear is the meaning of the latitudinal heat-flux component $F_{\theta}$. It is always poleward directed leading to the formation of warm polar caps - not in agreement with the results of Rieutord et al. (1994). We have shown with the simulations presented by the Figs. 8 and 9 how important this effect for the explanation of the solar/stellar differential rotation is. The meridional flow driven by the warm poles breaks the Taylor-Proudman theorem which would otherwise produce cylindrical isolines of the angular velocity which are not found by the the inversions of the helioseismology though. As we have also shown, the role of the radial eddy heat-flux $F_{r}$ is here of minor importance.

Acknowledgements. Axel Brandenburg (Copenhagen) is acknowledged for many discussions about the subject of this paper. L.L.K. is grateful to AIP for its hospitality and the visitor support.

\section{Appendix A: Rotating anisotropic turbulence}

The usual approach to rotating turbulences is to prescribe the turbulence for nonrotating fluid and then to derive the influence of rotation on the given original turbulence. As the first step in this procedure, we consider an incompressible fluctuating velocity field, $\boldsymbol{u}^{(0)}$, in a nonrotating fluid. The statistical properties

${ }^{1}$ In the top layer of the box where after Fig. 3 the polar value of $\left\langle u_{r}^{\prime 2}\right\rangle$ dominates the equatorial one also the polar value of the heatflux dominates its equatorial value.

${ }^{2} F_{\phi}$ is also negative at the southern hemisphere. of the original turbulence are given by its spectral tensor,

$$
\begin{aligned}
\hat{Q}_{i j}^{(0)}= & \frac{E(k, \omega)}{16 \pi k^{2}} K_{i j}(\boldsymbol{k}) \\
& +\frac{E_{1}(k, \omega)}{16 \pi k^{4}}\left(K_{i j}(\boldsymbol{G}) K_{m n}(\boldsymbol{G})-K_{i m}(\boldsymbol{G}) K_{j n}(\boldsymbol{G})\right) k_{m} k_{n},(\mathrm{~A} .1)
\end{aligned}
$$

where $\boldsymbol{G}$ is the radial unit vector, $\boldsymbol{k}$ is the wave vector, $\omega$ is frequency, and $K_{i j}$ is the projection tensor,

$K_{i j}(\boldsymbol{k})=\delta_{i j}-k_{i} k_{j} / k^{2}$.

Without rotation, the only preferred direction can be the radial one. Equation (A.1) allows for the radial anisotropy. Characteristic velocities are given by

$$
\begin{aligned}
& \left\langle u_{r}^{(0) 2}\right\rangle=\frac{1}{3} \int_{0}^{\infty} E(k, \omega) \mathrm{d} k \mathrm{~d} \omega, \\
& \left\langle u_{\mathrm{h}}^{(0) 2}\right\rangle-2\left\langle u_{r}^{(0) 2}\right\rangle=\frac{1}{3} \int_{0}^{\infty} E_{1}(k, \omega) \mathrm{d} k \mathrm{~d} \omega,
\end{aligned}
$$

where $u_{\mathrm{h}}^{(0)}$ is the horizontal velocity, $\left\langle u_{\mathrm{h}}^{(0) 2}\right\rangle=\left\langle u_{\phi}^{(0) 2}\right\rangle+\left\langle u_{\theta}^{(0) 2}\right\rangle$. Spectral functions must satisfy $E_{1} \geq-E$. A dimensionless anisotropy parameter, $a$, is introduced

$a=\left(\left\langle u_{\mathrm{h}}^{(0) 2}\right\rangle-2\left\langle u_{r}^{(0) 2}\right\rangle\right) /\left\langle u_{r}^{(0) 2}\right\rangle$

with $a \geq-1$ (Bochner's theorem). Negative $a$ means anisotropy of radial type with $\left\langle u_{\phi}^{(0) 2}\right\rangle=\left\langle u_{\theta}^{(0) 2}\right\rangle\left\langle\left\langle u_{r}^{(0) 2}\right\rangle\right.$ while positive $a$ means predominance of horizontal motions.

The rotational influence is involved via the tensor

$D_{i j}(\boldsymbol{\Omega})=\frac{\delta_{i j}+\frac{(2 \boldsymbol{k} \cdot \boldsymbol{\Omega})}{k^{2}\left(-\mathrm{i} \omega+v_{\mathrm{t}} k^{2}\right)} \epsilon_{i j p} k_{p}}{1+\frac{(2 \boldsymbol{k} \cdot \mathbf{\Omega})^{2}}{k^{2}\left(-\mathrm{i} \omega+v_{\mathrm{t}} k^{2}\right)^{2}}}$,

(Rüdiger 1989), which expresses the rotating turbulence in terms of the original one by the linear relation

$\hat{Q}_{i j}(\boldsymbol{k}, \omega)=D_{i m}(\mathbf{\Omega}) D_{j n}^{*}(\boldsymbol{\Omega}) \hat{Q}_{m n}^{(0)}(\boldsymbol{k}, \omega)$,

where the star here means complex conjugate and $v_{\mathrm{t}}$ is the effective viscosity by microscale turbulence.

With Eq. (A.6), the parameters of the rotating turbulence can be derived. We make, however, further simplifications by introducing the mixing-length approximation via a special form of the spectral functions

$E(k, \omega)=6 \cdot\left\langle u_{r}^{(0) 2}\right\rangle \delta\left(k-\ell_{\text {corr }}^{-1}\right) \delta(\omega), \quad E_{1}=a E$,

with $\ell_{\text {corr }}^{2} / v_{\mathrm{t}}=\tau_{\text {corr }}$ (Kitchatinov 1991), where $\ell_{\text {corr }}$ and $\tau_{\text {corr }}$ are the mixing length and time respectively. An advantage of Eq. (A.7) is that the effects of rotation can be expressed in terms of relatively simple parameters like the Coriolis number. The tensor (A.5) now reads

$D_{i j}(\mathbf{\Omega})=\frac{\delta_{i j}+\cos \vartheta \Omega^{*} \epsilon_{i j p} k_{p} / k}{1+\cos ^{2} \vartheta \Omega^{* 2}}$

where $\vartheta$ is the angle between the angular velocity and the wave vector. 
The relation (A.6) can now be integrated over the wave number space to find the one-point-correlation tensor. It reads

$$
\begin{aligned}
Q_{i j}= & \left\langle u_{r}^{(0) 2}\right\rangle\left\{\left(\phi\left(\Omega^{*}\right) \delta_{i j}+\phi_{\|}\left(\Omega^{*}\right) \frac{\Omega_{i} \Omega_{j}}{\Omega^{2}}\right)\right. \\
& +a\left(\phi^{\prime}\left(\Omega^{*}\right) \delta_{i j}+\phi_{1}\left(\Omega^{*}\right) \frac{(\boldsymbol{\Omega} \cdot \boldsymbol{G})^{2}}{\Omega^{4}} \Omega_{i} \Omega_{j}\right. \\
& +\phi_{2}\left(\Omega^{*}\right) G_{i} G_{j}-\phi_{3}\left(\Omega^{*}\right) \frac{(\boldsymbol{\Omega} \cdot \boldsymbol{G})}{\Omega^{2}}\left(\Omega_{i} G_{j}+\Omega_{j} G_{i}\right) \\
& \left.+\left(\phi_{\|}^{\prime}\left(\Omega^{*}\right) / 2+\phi_{3}\left(\Omega^{*}\right)\right)\left((\boldsymbol{\Omega} \cdot \boldsymbol{G})^{2} \delta_{i j}+\Omega_{i} \Omega_{j}\right) / \Omega^{2}\right) \\
& +a I_{0}\left(\Omega^{*}\right)\left(G_{i} \epsilon_{j m p}+G_{j} \epsilon_{i m p}\right) \frac{\Omega_{m}}{\Omega_{p}} G_{p} \\
& \left.+a I_{1}\left(\Omega^{*}\right) \frac{(\boldsymbol{\Omega} \cdot \boldsymbol{G})}{\Omega^{3}}\left(\Omega_{i} \epsilon_{j m p}+\Omega_{j} \epsilon_{i m p}\right) \Omega_{m} G_{p}\right\}
\end{aligned}
$$

for rotating anisotropic turbulence. For slow rotation

$\phi \simeq 1, \phi^{\prime} \simeq 1 / 2, \phi_{2} \simeq-1 / 2, I_{0} \simeq 2 \Omega^{*} / 5$,

$\left(\Omega^{*} \ll 1\right.$, other functions are of second or of higher order in $\left.\Omega^{*}\right)$, and for fast rotation $\left(\Omega^{*} \gg 1\right)$

$\phi=\phi_{\|} \simeq \frac{3 \pi}{8 \Omega^{*}}, \phi^{\prime} \simeq \frac{3 \pi}{64 \Omega^{*}}, \phi_{\|}^{\prime} \simeq \phi_{2} \simeq \phi_{3} \simeq \frac{3 \pi}{32 \Omega^{*}}$,

$\phi_{1} \simeq \frac{9 \pi}{64 \Omega^{*}}, I_{0} \simeq \frac{3 \pi}{16 \Omega^{* 2}}, I_{1} \simeq \frac{9 \pi}{16 \Omega^{* 2}}$

$\left(\Omega^{*} \gg 1\right)$. Full expressions for the functions are too bulky to reproduce here. Some of them can be found elsewhere (Kitchatinov et al. 1994; Kitchatinov 2004).

For the off-diagonal component, $Q_{\theta r}$ yields

$$
\begin{aligned}
Q_{\theta r}= & -\sin \theta \cos \theta\left\langle u_{r}^{(0) 2}\right\rangle\left(\phi_{\|}\left(\Omega^{*}\right)\right. \\
& \left.+a\left(\phi_{\|}^{\prime}\left(\Omega^{*}\right) / 2+\cos ^{2} \theta \phi_{1}\left(\Omega^{*}\right)\right)\right) .
\end{aligned}
$$

$Q_{\theta r}$ is thus always negative.

The sign of the off-diagonal component $Q_{\phi r}$ of the correlation tensor is only controlled by the turbulence anisotropy, i.e.

$Q_{\phi r}=\left\langle u_{r}^{(0) 2}\right\rangle a \sin \theta\left(I_{0}\left(\Omega^{*}\right)+\cos ^{2} \theta I_{1}\left(\Omega^{*}\right)\right)$.

$Q_{\phi r}$ is thus negative for radial-type of anisotropy $(a<0)$. It vanishes for isotropic turbulence $(a=0)$ and is one of the known components of the $\Lambda$-effect.

With the mixing-length approximation (A.7), the relation between the turbulent thermal conductivity and the velocity correlation tensor becomes

$\chi_{i j}=\tau_{\text {corr }} Q_{i j}=\tau_{\text {corr }} \int \hat{Q}_{i j}(\boldsymbol{k}, \omega) \mathrm{d} \boldsymbol{k} \mathrm{d} \omega$.
Note the factor 2 by which this relation differs from Eq. (14) which only holds in the limit $\chi \rightarrow 0$.

\section{References}

Belvedere, G., Paternò, L., \& Stix, M. 1980, Geophys. Astrophys. Fluid Dyn., 14, 209

Canuto, V. M., Minotti, F. O., \& Schilling, O. 1994, ApJ, 425, 303

Canuto, V. M., \& Minotti, F. 2001, MNRAS, 328, 829

Canuto, V. M. 2002, MNRAS, 337, 713

Chan, K. L. 2001, ApJ, 548, 1102

Durney, B. R., \& Roxburgh, I. W. 1971, Sol. Phys., 16, 3

Duvall, T. L., \& Gizon, L. 2000, Sol. Phys., 192, 177

Giesecke, A., Ziegler, U., \& Rüdiger, G. 2004, Phys. Earth Planet. Int., submitted

Gough, D. O. 1978, The estimation of fluxes due to small scale turbulences, in Workshop on Solar Rotation (Catania), 337

Hall, D. S. 1991, Learning about the stellar dynamos from long-term photometry of starspots, in The Sun and Cool Stars: Activity, Magnetism, Dynamos, ed. I. Tuominen, D. Moss, \& G. Rüdiger (Berlin: Springer), 353

Käpylä, P. J., Korpi, M. J., \& Tuominen, I. 2004, A\&A, 422, 793

Kitchatinov, L. L. 1991, A\&A, 243, 483

Kitchatinov, L. L., \& Rüdiger, G. 1993, A\&A, 276, 96

Kitchatinov, L. L., Pipin, V. V., \& Rüdiger, G. 1994, Astron. Nachr., 315,157

Kitchatinov, L. L., \& Rüdiger, G. 1999, A\&A, 344, 911

Kitchatinov, L. L. 2004, Astron. Rep., 48, 153

Küker, M., Rüdiger, G., \& Kitchatinov, L. L. 1993, A\&A, 279, 1

Küker, M., \& Stix, M. 2001, A\&A, 366, 668

Messina, S., \& Guinan, E. F. 2003, A\&A, 409, 1017

Reiners, A., \& Schmitt, J. H. M. M. 2003, A\&A, 398, 647

Reiners, A., \& Schmitt, J. H. M. M. 2003, A\&A, 412, 813

Rieutord, M., Brandenburg, A., Mangeney, A., \& Drossart, P. 1994, A\&A, 286, 471

Rüdiger, G. 1989, Differential Rotation and Stellar Convection: Sun and Solar-type Stars (New York: Gordon \& Breach Science Publishers)

Rüdiger, G., Tschäpe, R., \& Kitchatinov, L. L. 2002, MNRAS, 332, 435

Rüdiger, G., \& Hollerbach, R. 2004, The Magnetic Universe (Berlin: Wiley-VCH)

Spiegel, E. A., \& Zahn, J. P. 1992, A\&A, 265, 106

Tilgner, A., \& Busse, F. H. 1997, J. Fluid Mech., 332, 359

Weiss, N. O. 1965, The Observatory, 85, 37

Ziegler, U. 1998, Comput. Phys. Commun., 109, 111

Ziegler, U. 1999, Comput. Phys. Commun., 116, 65 